\title{
Teratological Effects of Retinoids on Skeletal Elements of Swiss Albino Mice
}

\author{
Dr Rashmi Sharma ${ }^{1}$ Ashok Gupta ${ }^{2}$ \\ Department Of Zoology Spc Gca M.D.S.U. Ajmer Rajasthan India
}

\begin{abstract}
The specific branch o developmental biology which deals with abnormalities caused as a result of failure of normal pattern of development is referred as teratogenesis. Retinoids are essential for spermatogenesis, oogenesis, placental development, foetal morphogenesis and growth. The role of vitamin A in vision is well known. Active derivative of vitamin A (Retinoids) play an important and multiple role in Mammalian development and homeostasis (Sapin, et.al. 1997, Dup et al 1997). These essential dietary compounds are needed in very small quantities, they are not synthesized by the animals and are obtained from external sources in the form of $\beta$ carotene (C40H56). In cells of intestine $\beta$ carotene is converted into vitamin $A$ alcohol and is transported by blood to liver where it is esterfied and stored as vitamin A palmitate. RA is not stored in liver but is derived from retinal and it is biologically the most potent form of vitamin A. Vitamins are organic compounds which are devided into 2 categories $i$ Fat soluble $(A, D, E, K)$ and ii water soluble $(B$ and $C)$. Vitamin A belongs to fat soluble category of vitamins. A fat soluble substance essential for life was first discovered in egg yolk in 1909 by Stepp (Pawson, 1981; Robert and Sporn, 1984). It was confirmed by Mc Collum Devis in 1913 when he found a compound with similar biological activity in butter fats, egg yolk and cod liver oil and was named fat soluble vitamin A. The development of an organism is a complex process of embryogenesis involving cell proliferation, differentiation, migration and organogenesis. Many agents interfering the development process can cause malformations in the embryo. The study of these congenital abnormalities is called teratology and agents which are responsible for causing these malformations are called teratogens. Susceptibility to teratogens depends on the genotype of the organism, including species as well as strain differences. Etienne Geoffrey Saint Hilaire and his son in 1820 started experiments on chick embryo by disturbing its environment in different ways at different embryonic stages. They found some anomalies like Trioncephally, atrophy of eyes and spina bifida in his experiments (Tuli, 1968). In 1877 Dareste reported found some anomalies like Trioncephally, atrophy of eyes and spina bifida in his Experiments (Tuli, 1968). 4mg RA severely damaged skin on later stage ie 11th and 14th day gestation period, of development, shows disruption and folds of skin look like over verying skin flaps. In the present paper effects of different doses of vitamin A on Skeletal elements on different development stages of swiss albino mice are studied.
\end{abstract}

Keywords: Retinoids, Teratology, Swiss albino mice, Skeletal elements.

\section{Introduction}

The branch of development biology which deals with abnormalities of development or failure of normal development is referred as teratology. Retinoids are essential for spermatogenesis oogenesis, placental development, foetal morphogenesis and growth. The role of vitamin A in vision is well known. Active derivative of vitamin A (Retinoids) play an important and multiple role in Mammalian development and homeostasis (Sapin, et.al. 1997, Dup et al 1997). These essential dietary compounds are needed in very small quantities, they are not synthesized by the animals and are obtained from external sources in the form of $\beta$ carotene ( $\mathrm{C} 40 \mathrm{H} 56)$. In cells of intestine $\beta$ carotene is converted into vitamin A alcohol and is transported by blood to liver where it is esterified and stored as vitamin A palmitate. RA is not stored in liver but is derived from retinal and it is biologically the most potent form of vitamin A. Vitamins are organic compounds which are devided into 2 categories

1. Fat soluble $(\mathrm{A}, \mathrm{D}, \mathrm{E}, \mathrm{K})$

2. Water soluble (B and C).

Vitamin A belongs to fat soluble category of vitamins. A fat soluble substance essential for life was first discovered in egg yolk in 1909 by Stepp (Pawson, 1981; Robert and Sporn, 1984). It was confirmed by Mc Collum Devis in 1913 when he found a compound with similar biological activity in butter fats, egg yolk and cod liver oil and was named fat soluble vitamin A. The name of vitamin was given by Dummond In 1920. The natural and synthetically obtained vitamin $A$ is found in several forms of alcohol (Retinol), aldehyde (Retinol palmitate), acetate and retinoic acid. They were collectively known as retinoids. 
Retinol or alcoholic form of Vitamin $A$ is a unstable organic compound. They are easily oxidized specially in the presence of heat. The ester form of vitamin A are fairly stable. When vitamin $\mathrm{A}$ is taken in the form of esters (palmitate/acetate), its converted into alcoholic form (Retinol) in the intestinal cells to be carried to liver, where it is converted palmitate form for storage. Retinoic acid is not stored in the liver but it is derived from retinol and it is biologically most potent form. The development of an organism is a complex process of embryogenesis involving cell proliferation, differentiation, migration and organogenesis. Many agents interfering the development process can cause malformations in the embryo. The study of these congenital abnormalities is called teratology and agents which are responsible for causing these malformations are called teratogens. Susceptibility to teratogens depends on the genotype of the organism, including species as well as strain differences.

Teratological experiments was started from 1820s with the studies of Etienne Geoffrey Saint Hilaire and his son on chick embryo by disturbing its environment in different ways at different embryonic stages. They found some anomalies like Trioncephally, atrophy of eyes and spina bifida in his Experiments (Tuli, 1968). In 1877 Dareste reported some congenital malformation in the chick embryo by environmental disturbance.

\section{Materials and Methods}

The mice were obtained from mice breeding center, Department of Zoology M.D.S.U. Ajmer. Animals were fed synthetic diet mice feed pallets (Brook Bond Lipton India Ltd.)Supplemented with germinated grains, seasonal green vegetables, multivitamin drops and water ad libitum. Tetracycline mixed in water was regularly given. There are four virgin female mice 25+-1gm caged with fertile healthy male in the evening and these were examined for presence of vaginal plug, the next morning. The day appearance of vaginal plug was considered as day 0 of pregnancy.

\section{The veginal plug}

After copulation a secretion of seminal vesicles in the ejaculate of the male coagulate to form a plug in the vegina extending from the cervix to the valve, where it is ordinarily visible and is a convenient external sign that mating has occurred (Green, 1996).

\section{Duration of pregnancy}

The gestation period in this species is 19 days and occasionally 20 days. The youngs are most frequently born in the early hours of the morning between midnight and 4 AM.

\section{Objectives}

Investigation of the effects of retinoic acid on different developmental stages $\left(5^{\text {th }}, 8^{\text {th }}, 11^{\text {th }}\right.$, and $14^{\text {th }}$ ) of mouse embryo.To study effects of RA on organogenesis of skin, liver, heart .

\section{Effect of RA on skeletal elements.}

Experimental design

The pregnant females were devided into following groups:

(6 animals per group )

Group A : untreated

Group B : Treated

Doses

Suitable (non lethal or sublethal) doses of RA were screened on mice embryos on different stages of developments. The following doses were found to produce various teratological defects on developing mice embryos

1. 2mg RA /pregnant female.

2. $4 \mathrm{mg}$ RA /pregnant female.

\section{Effects of RA on Skeletal elements}

\section{Results and Discussions}

The analysis of skeletal malformation caused by RA was carried out by in toto alizarin and Victoria blue staining technique. Ra effect on length of skeletal elements, increased or decreased number of bones, ab shape of bones, abnormal enlargement of bones, and degree of ossification. 


\section{Teratological Ranks}

The normal skeletal elements is designated as $\mathrm{T} 0$ rank. Where as the increasing teratogenecity is ranked from $\mathrm{T} 1, \mathrm{~T} 2, \mathrm{~T} 3, \mathrm{~T} 4$ and $\mathrm{T} 5 . \mathrm{T} 0$ : Teratological rank normal pattern formation of skeletal element without any deformities in such cases skeletal elements almost completely ossified.

T1 : This is lowest degree. Lesser degree of ossification is observed.The extent of ossification has been defined in terms of ++++ .

$4+$ indicates highest degree of ossification and only single + indicates very poor ossification.

T2 : These cases show slightly lesser degree of ossification and the bone length is more elongated.

T3: In these cases the gap between the long bones is increased.

T4: Ossification is almost ab Bones are elongated and gap between the long bones increased.

T 5: Change in number of bones and ossification absent.

Untreated (Control).

The untreated freshly delivered embryos stained in toto for sk ele showed organization of skeletal elements of various parts of the body.

\section{Head Region Bones}

Frontal, Parietal, nasal, premaxillary and mandibular bones. The other important bones include bassioccipital, exoccipital.

Trunk and Girdle bones

Trunk region bears ribs, sternum, scapula, clavicle,pelvic girdle, thoracic, lumber and sacral.

\section{Limb bones}

Fore limb bones are humerus, radius-ulna, carpals, metacarpals. In hind limb femur, tibia fibula and tarsal elements.

\section{Treated $2 \mathrm{mg}$ RA on $5^{\text {th }}$ day gestation}

Dose was given to the pregnant mice on $5^{\text {th }}$ day gestation. This drug did not effect the Skeletal elements of the embryo. The skeletal pattern was almost similar to that of control, untreated.

\section{2mg RA on $8^{\text {th }}$ day gestation}

The number of ribs, sternum and caudal vertebrae were also similar to untreated control embryo. The analysis of fore and hind limb sk ele of RA treated cases does not indicate teratological effects. However the metacarpals are slightly elongated. In these bones the ranking of teratological effects was $\mathrm{T} 1$.

\section{$2 \mathrm{mg}$ RA on $11^{\text {th }}$ day gestation}

The teratogenecity was not observed to be severe.Degree of ossification was reduced particularly in cranial and facial bones.The number of ribs and sternum was same as it is found in control.In the fore limb humerus and in hind limb femur were similar to the control. The teratogenic effects was observed basically in tibia fibula and radius ulna. In these bones gap between radius ulna in the fore limb and between tibia fibula of hind limb is slightly increased. These bones were longer than the bones observed in untreated.Such cases are designated as T4 and T5 in teratogenic ranking. It was interesting to note that ossification is of lower degree (+ ranking) in these bones. When fore limb compared with hind limb showed ossification of lesser degree. As compared to fore limb.

\section{2mg RA on $14^{\text {th }}$ day gestation}

The cranial skeletal elements showed poor alizarin red staining, indicating poor ossification .This dose did not affect the number of ribs and sternum and their arrangement. In the fore limb the radius ulna are slightly elongated and the gap between radius ulna was wider as compared to control. The metacarpals were elongated and their gap increased such cases were designated as T1 and T3 in teratogenic ranking. 


\section{4mg RA on $5^{\text {th }}$ day gestation}

In these cases skeletal elements of head region thoracic region, lumbar region, caudal region and ribs are similar to untreated cases.

\section{4mg RA on $8^{\text {th }}$ day gestation}

Showed normal sk ele in the cephalic region except slightly reduced ossification of cranial bones. Similarly ossification is found to be reduced in fore limb and hind limb bones. The gap between radius and ulna was increased. Phalanges metacarpals and carpals are slightly elongated. In the hind limb also degree of ossification is reduced in bones and gap is increased.

\section{$4 \mathrm{mg}$ RA on $11^{\text {th }}$ day gestation}

The skeletal elements of treated cases were not ossified Facial bones showed alizarin red staining and cranial bones showed Victoria blue staining. In such cases bones of cranial region are elongated and cleft palate is reduced in size. Thoracic bonesi.e. ribs, sternum and respective vertebrae showed positive staining with alizarin red $\mathrm{S}$. When caudal region is stained with alizarinVictoria blue double staining method caudal vertebrae are not distinct or absent in such cases.

Skeletal elements of fore limbs humerus, radius ulna and digits are found to be arranged in sequence.

Humerus shows positive staining with alizarin but radius ulna shows positive staining with Victoria blue. In these cases fore limb skeletal elements radius ulna are not only elongated but also noticeable gap is observed between these two bones. Distal phalanges skeletal elements also show positive staining with Victoria blue indicating cartilaginous skeletal elements. Bones of hind limb are also affected with this dose of RA. Ossification percent is slightl reduced in femur. In these cases not only tibia fibula shows enhanced growth but femur also indicate increased growth. The gap between tibia and fibula further increased. In the caudal region tail vertebrae are not distinct.

\section{4mg RA on $14^{\text {th }}$ day gestation}

In these cases in the cephalic region cranial and facial bonesare abnormal and slightly elongated. There is obvious cleft palate defects in these cases. In thoracic region the pattern fformation of bones is almost similar the control except Reduced degree of ossification. Fore limb skeletal elements are clearly observed with alizarin but some bones such as radius and ulna and other distal bones are elongated. There is clear indication of widening of gap between radius ulna with more elongation, less ossification as compared to untreated control.

Hind limb skeletal elements of $4 \mathrm{mg} \mathrm{RA}$ treated cases shows reduced ossification in the long bone. The gap between tibia and fibula is more than the untreated cases. The bones of distal most region of hind limb also showed elongation and lesser degree of ossification. In the caudal region the number of vertebrae was found to be normal except lesser degree of ossification.(Teratogenic ranking is $(-+)$.

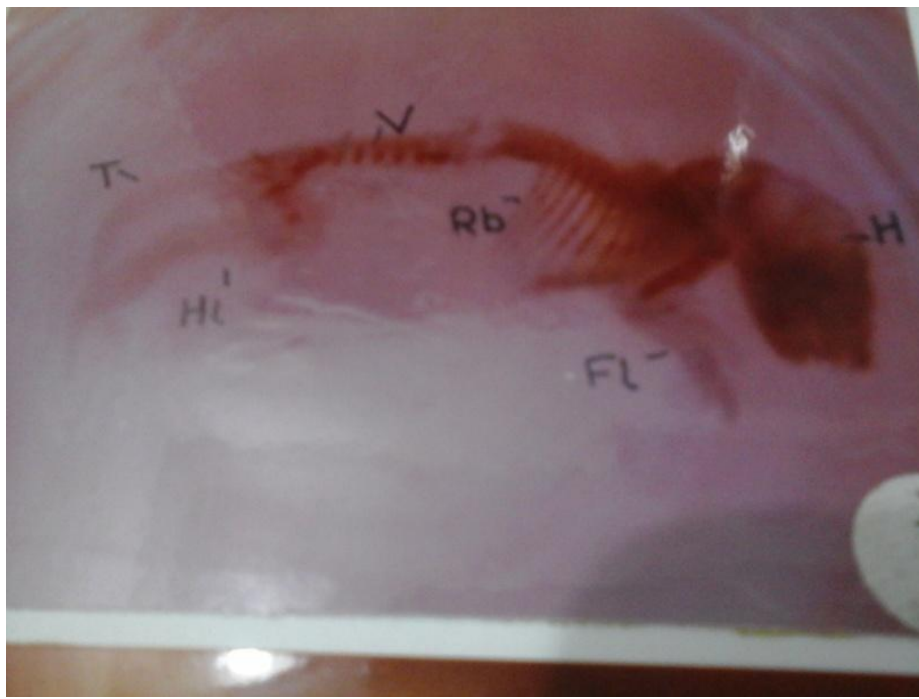

Fig1 : Showing arrangement of skeletal elements of head, thoracic and lumber region; reduced degree of ossification and absent of caudal vertebrae. 


\section{Conclusion and Summary}

RA causes skeletal malformations when treatment is given on $8^{\text {th }}, 11^{\text {th }}$, and $14^{\text {th }}$ day gestation. $5^{\text {th }}$ day gestation female when treated with $2 \mathrm{mg}$ and $4 \mathrm{mg}$ concentration RA does not produce any effect on the skeletal elements. RA treatment produces mild or moderate type of teratological defectswhich include Shorting or elongation of bones, increasing gap between bones of zeugopodium of skeletal elements. In most cases skeletal elements were cartilaginous, which indicate inhibition of the process of ossification by RA. The retinoids on foetal rat bones in culture are reported to be acting by inducing release of Proteoglycans followed by cartilaginous breakdown (Fell and Mellanby , 1953; Kistler ,1978,82) Gallandra et.al.(1980) demonstrated that RA induce cartilage resorption require RNA,Protein and glycoprotein synthesis and specifically changes the protein synthesis pattern. These finding suggest that in cartilaginous RA may exert its action by altering gene expression.

Retinoids have been found to inhibit or delay condrogenesis was evident at both the dose of RA used i.e. $2 \mathrm{mg}$ and $4 \mathrm{mg} /$ pregnant female. Retinoids also inhibit or delay mesenchyme differentiation during amphibian limb regeneration (Sharma, 1982; Maden, 1983; Sharma and Anton, 1986; Sharma and Niazi, 1988;Niazi et.al.1989; Scadding 1989). Disorganization of chondrocytes and changes in extra cellular matrix have been noted in the leg and wing bud of RA treated embryos. Sharma and Anton (1986 a ) found that oriented collagen fibrils in the extracellular matrix was absent in the Retinol palmitate treated blastema of axolotals This characteristic feature of ECM is observed during redifferentiation of blastemal mesenchymel cells of untreated (control) axolotls during prechondrogenic condensation. It has also been demonstrated that when culture medium was supplemented with high concentration $\left(10^{-5} \mathrm{~m}\right)$ of RA , chondrogenesis of limb bud was inhibited (Pannyacler., et.al. 1978; Lewis., et.al. 1978; Pignatello., et.al.1997).

\section{References}

[1]. Coblan, S.Q.(1953):Excessive intake of Vit A as a cause of congenital anomalies in Rat. Science 117:535-536.

[2]. Coblan, S.Q. (1954):Congenital anomalies in rat produced by excessive intake of vit A during pregnancy.Pediatrics. 13 556-557.

[3]. Dickman, E.D. and Smith, S.M. (1996) Selective regulation of cardiomyocyte expressionand cardiac morphogenesis by RA dev dynamics 206(1):39-48.

[4]. Effendy. I., (1996): Differential irritant skin responses to topical RA and sodium layryl sulphate alone and in crossover design British J. of Dermatology 134: 420-430. (Abstract).

[5]. Elmzar, M.M.A.(1996). Pattern of Retinoid induced teratogenic effect. Possible relationship with relative selectivity for nuclear retinoid receptors RAR-alpha,RAR-beta and RAR gama. Teratology53(3):158-167.(Abstract).

[6]. Fisher, G.J. and Voiegwwa, J.J. (1996): molecular mechanisms of retinoid actions in skin,FASEBJ.10(9).1002-1013.

[7]. Giroud, R.and Martinet,(1954): Fertes du Polasis chezl embryos de rat per hypervitaminoseA., Comp.Rend.Soc.Biol.148:17421743.

[8]. Goodman, A.B. (1996) : Congenital anomalis in relative of Schizophrenic probands may indicate a retinoid pathology. Schizophrenia Research 19: 2-3:169-170.(Abstract).

[9]. Griffiths. C. E. M. (1996): Tropical RA changes the epidermal cell surface glycosylation pattern towards that of a mucosa epithelium. British Journal of Dermatology. 134; 431-436.

[10]. Leelaprute. L ; Boonpucknaving. V.; Bharmar apratuati. N., and Weerapradist. W.(1973).

[11]. Hypervitaminosis A in rat Arch. Sci 85:42-55.

[12]. Kalter, H.C.(1960): The teratogenic effect of hypervitaminosis upon the face and mice of inbred mice. Ana. N.Y. Acad. Sci.85:42-55.

[13]. Kalter, H.C. and Warkany J. (1961): Experimental production of congenital malformation In strains of inbred mice by maternal treatment with hypervitaminosis. Amer J. path,381-21.

[14]. Kochhar, D.M. (1967): Teratogenic activity of Retinoic Acid. Acta Pathol Micro. Biol. Scand. 70:398-404.

[15]. Moore, T. (1957) : "Vitamin A" Am. Elsevier, New Delhi.

[16]. Niazi, I.A. : Pescitelli M.J.; and Stocum, D.L., (1985); Stage dependent effects of RA on regenerating urodele limbs. 